\title{
CAMA
}

Centre for Applied Macroeconomic Analysis

\section{Efficient Jacobian evaluations for estimating zero lower bound term structure models}

\section{CAMA Working Paper 77/2013 December 2013}

\section{Leo Krippner}

Reserve Bank of New Zealand and

Centre for Applied Macroeconomics Analysis

\section{Abstract}

Faster extended Kalman filter estimations of zero lower bound models of the term structure are possible if the analytic properties of the Jacobian matrix for the measurement equation are exploited. I show that such results are straighforward to incorporate, at least in Monte-Carlo-based implementations, and that will facilitate fast and robust estimations of zero lower bound term structure models with the iterated extended Kalman filter. 


\section{Keywords}

Black framework, zero lower bound, shadow short rate, term structure model

\section{JEL Classification}

C18, E43, G12

\section{Address for correspondence:}

(E) cama.admin@anu.edu.au

The Centre for Applied Macroeconomic Analysis in the Crawford School of Public Policy has been established to build strong links between professional macroeconomists. It provides a forum for quality macroeconomic research and discussion of policy issues between academia, government and the private sector.

The Crawford School of Public Policy is the Australian National University's public policy school, serving and influencing Australia, Asia and the Pacific through advanced policy research, graduate and executive education, and policy impact. 


\title{
Efficient Jacobian evaluations for estimating zero lower bound term structure models
}

\author{
Leo Krippner*
}

6 December 2013

\begin{abstract}
Faster extended Kalman filter estimations of zero lower bound models of the term structure are possible if the analytic properties of the Jacobian matrix for the measurement equation are exploited. I show that such results are straighforward to incorporate, at least in Monte-Carlo-based implementations, and that will facilitate fast and robust estimations of zero lower bound term structure models with the iterated extended Kalman filter.
\end{abstract}

JEL: C18, E43, G12

Keywords: Black framework; zero lower bound; shadow short rate; term structure model

\section{Introduction}

This article shows that faster extended and iterated extended Kalman filter estimations of Black (1995) zero lower bound (ZLB) models of the term structure are possible if the analytic properties of the Jacobian matrix for the measurement equation are exploited. ${ }^{1}$

As background, interest rates for multi-factor Black (1995) models do not have closed form analytic solutions. Hence, calculating a set of model yields for a given set of state variables at time $t$ and the parameters within a Black (1995) model, what I will call an implementation, necessarily requires numerical methods. For example, Black (1995) models using Gaussian affine term structure models to represent the shadow term structure (hereafter B-GATSMs) have been implemented with finite-difference grids, interest rate lattices, and Monte Carlo simulations; Kim and Singleton (2012), Richard (2013), and Bauer and Rudebusch (2013) are recent respective examples. Recent advances in Priebsch (2013) and Krippner (2013a) offer faster B-GATSM implementations, respectively via a close second-order approximation evaluated with numerical methods, and a Monte Carlo simulation with a control variate. The control variate is itself an alternative

\footnotetext{
${ }^{*}$ Reserve Bank of New Zealand and Centre for Applied Macroeconomic Analysis. Email: leo.krippner@rbnz.govt.nz.

${ }^{1}$ The terminology "zero lower bound/ZLB" is standard in the literature, even though a non-zero lower bound may be appropriate in practice to accommodate central bank policy rate preferences and/or institutional frictions; see Jarrow (2013) on the latter. Non-zero lower bounds can readily be incorporated into ZLB models; e.g. see Krippner (2013c) p. 5 or Wu and Xia (2013) eq. 1.
} 
shadow/ZLB-GATSM framework proposed in Krippner (2012, 2013), which I hereafter call the K-GATSM. ${ }^{2}$

When estimating B-GATSMs, implementations will be required for each observation of yield curve data, and the complexity is further compounded by shadow/ZLB-GATSM measurement equations having a non-linear dependence on the shadow-GATSM state variables. Hence, B-GATSM estimations often employ the extended Kalman filter (EKF) with numerically evaluated Jacobian matrices at each observation to calculate the value of the log-likelihood function for a given set of parameters; e.g. see Kim and Singleton (2012) and Bauer and Rudebusch (2013) p. 10 as referenced earlier. ${ }^{3}$ Such estimations are therefore very time consuming given $N+1$ implementations or $2 N+1$ implementations (where $N$ is the number of factors) are required respectively for a first-difference or central-difference Jacobian approximation. In addition, because each implementation of a B-GATSM is subject to numerical approximation error, taking differences between implementations to obtain the Jacobian elements numerically could result in significant error magnification.

Fortunately, I show that it is easy to exploit the analytic properties of the B-GATSM measurement equation to obtain the Jacobian using the numerical implementations already undertaken for the central value of the state variables. That principle has already been exploited for K-GATSMs in Krippner (2013c) and Wu and Xia (2013), as I briefly illustrate in section 3 after outlining the appropriate notation for shadow/ZLB-GATSMs in section 2. In section 3, I first show that the same principle holds for the generic B-GATSM, and then illustrate how that result applies in practice to the estimation of B-GATSMs implemented with Monte Carlo methods. I conclude in section 5, noting that the IEKF estimations with analytic Jacobians are likely to be preferable to the EKF or unscented Kalman filter for B-GATSM estimations.

\section{Shadow/ZLB-GATSMs and estimation}

I adopt the generic GATSM specification from Dai and Singleton (2002) pp. 437-38 to define the shadow-GATSM. Hence, the shadow short rate is:

$$
\mathrm{r}(t)=a_{0}+b_{0}^{\prime} x(t)
$$

where $a_{0}$ is a constant, $b_{0}$ is a constant $N \times 1$ vector containing the weights for the $N$ state variables $x_{n}(t)$, and $x(t)$ is an $N \times 1$ vector containing the $N$ state variables $x_{n}(t)$. Under the physical $\mathbb{P}$ measure, $x(t)$ evolves as the following correlated vector Ornstein-Uhlenbeck process:

$$
\mathrm{d} x(t)=\kappa[\theta-x(t)] \mathrm{d} t+\sigma \mathrm{d} W(t)
$$

where $\theta$ is a constant $N \times 1$ vector representing the long-run level of $x(t), \kappa$ is a constant $N \times N$ matrix that governs the deterministic mean reversion of $x(t)$ to $\theta, \sigma$ is a constant

\footnotetext{
${ }^{2}$ The $\mathrm{Wu}$ and Xia (2013) model is a discrete-time version of the K-GATSM, although it is derived differently. K-GATSMs are much quicker to implement than B-GATSMs, because they only require univariate numerical integration regardless of the number of factors, and they have been shown to give an acceptable approximation to B-GATSMs in practice; see Krippner (2013c), Christensen and Rudebusch (2013a, b), and Wu and Xia (2013).

${ }^{3}$ Kim and Singleton (2012) method confirmed by personal correspondence with the authors. Richard (2013) evaluates the log-likelihood function with a derivative-free method numerical methods, but repeated implementations are still required at each observation.
} 
$N \times N$ matrix representing the potentially correlated volatilities of $x(t)$, and $\mathrm{d} W(t)$ is an $N \times 1$ vector with independent Wiener components $\mathrm{d} W_{n}(t) \sim \mathrm{N}(0,1) \sqrt{\mathrm{d} t}$.

The market prices of risk are linear with respect to the state variables, i.e.: ${ }^{4}$

$$
\Pi(t)=\sigma^{-1}[\gamma+\Gamma x(t)]
$$

where $\gamma$ and $\Gamma$ are respectively a constant $N \times 1$ vector and constant $N \times N$ matrix. Under the risk-adjusted $\mathbb{Q}$ measure, the process for $x(t)$ is:

$$
\mathrm{d} x(t)=\tilde{\kappa}[\tilde{\theta}-x(t)] \mathrm{d} t+\sigma \mathrm{d} \tilde{W}(t)
$$

where $\tilde{\kappa}=\kappa+\Gamma$ and $\tilde{\theta}=\tilde{\kappa}^{-1}(\kappa \theta-\gamma)$.

For the purposes of the brief discussion of K-GATSMs in section 3, it is sufficient to note that the system described above produces closed form analytic solutions for shadow forward rates $\mathrm{f}[x(t), \mathbb{A}, u]$ and annualized option volatilities $\omega[\mathbb{A}, u]$, as a function of the state variables $x(t)$, the parameter set $\mathbb{A}=\{\kappa, \theta, \sigma, \gamma, \Gamma\}$, and the time to maturity $u$ (see Krippner (2013c) pp. 14-15 for details). For B-GATSMs, the path of the shadow short rate under the risk-adjusted $\mathbb{Q}$ measure, $\mathrm{r}(t+u)$, may be obtained from the solution to equation 4 (e.g. see Meucci (2010) p. 3), i.e.:

$$
x(t+u)=\tilde{\theta}+\exp (-\tilde{\kappa} u)[x(t)-\tilde{\theta}]+\int_{t}^{t+u} \exp (-\tilde{\kappa}[u-v]) \sigma \mathrm{d} W(v)
$$

which gives:

$$
\mathrm{r}(t+u)=a_{0}+b_{0}^{\prime}\left\{\tilde{\theta}+\exp (-\tilde{\kappa} u)[x(t)-\tilde{\theta}]+\int_{t}^{t+u} \exp (-\tilde{\kappa}[u-v]) \sigma \mathrm{d} W(v)\right\}
$$

Regarding the estimation of shadow/ZLB-GATSMs, the state equation is that of the shadow-GATSM, i.e. the linear expression:

$$
x_{t+1}=\theta+\exp (-\kappa \Delta t)\left(x_{t}-\theta\right)+\varepsilon_{t+1}
$$

where $\Delta t$ is the time increment between observations, the subscript $t$ is an integer index for the time series of term structure observations, and $\varepsilon_{t+1}$ is the $N \times 1$ vector of innovations to the state variable vector $x_{t+1}$.

The measurement equation for both the K-GATSM and B-GATSM may be represented as:

$$
\underline{\mathrm{R}}_{t}=\underline{\mathrm{R}}\left[x_{t, i}, \mathbb{A}\right]+\eta_{t}
$$

where $\underline{\mathrm{R}}_{t}$ is the $K \times 1$ vector of interest rate data $\underline{\mathrm{R}}\left[t, \tau_{k}\right]$ for the $K$ maturities at time index $t, \eta_{t}$ is the $K \times 1$ vector of components unexplained by the shadow/ZLB-GATSM, and $\underline{\mathrm{R}}\left[x_{t, i}, \mathbb{A}\right]$ is the $K \times 1$ vector of shadow/ZLB-GATSM rates $\underline{\mathrm{R}}\left[x_{t, i}, \tau_{k}\right]$ for each maturity. The latter are obtained from an implementation of either the K-GATSM or B-GATSM, where $x_{t, i}$ is the estimate of the state variable vector at time index $t$ and iteration $i$ of the IEKF (the EKF sets $i=0$ ). The K-GATSM uses interest rates defined by equation 11 in section 3, while the B-GATSM uses interest rates defined by equation 14 in section 4 .

\footnotetext{
${ }^{4}$ This is the "essentially affine" specification from Duffee (2002), but for a model with full Gaussian dynamics. Also see Cheridito, Filipović, and Kimmel (2007) for further discussion on market price of risk specifications.
} 
Estimating the K-GATSM or B-GATSM via the EKF or IEKF requires the $K \times N$ Jacobian matrix $H_{t, i}$ for $\underline{\mathrm{R}}\left[x_{t, i}, \mathbb{A}\right]$. Omitting the parameter dependence hereafter and concentrating on a single maturity $\tau_{k}$, each row of $H_{t, i}$ is defined as:

$$
H_{t, i, k}=\left.\frac{\partial}{\partial x(t)} \underline{\mathrm{R}}\left[x(t), \tau_{k}\right]\right|_{x(t)=x_{t, i}}
$$

I provide $H_{t, i, k}$ for the K-GATSM in section 3 and derive $H_{t, i, k}$ for the B-GATSM in section 4 .

\section{K-GATSM term structures and Jacobian}

K-GATSM forward rates are defined as (see Krippner (2013c) p. 16):

$$
\underline{\mathrm{f}}[x(t), u]=\mathrm{f}[x(t), u] \cdot \Phi\left[\frac{\mathrm{f}[x(t), u]}{\omega[u]}\right]+\omega(u) \cdot \phi\left[\frac{\mathrm{f}[x(t), u]}{\omega[u]}\right]
$$

where $\Phi[\cdot]$ and $\phi[\cdot]$ are respectively the cumulative normal and marginal normal density functions. The interest rate for a given maturity $\tau_{k}$ is obtained using the standard term structure relationship: ${ }^{5}$

$$
\underline{\mathrm{R}}\left[x(t), \tau_{k}\right]=\frac{1}{\tau_{k}} \int_{0}^{\tau_{k}} \underline{\mathrm{f}}[x(t), u] \mathrm{d} u
$$

In practice, calculating $\underline{\mathrm{R}}\left[x_{t, i}, \tau_{k}\right]$ for a given state variable vector $x_{t, i}$ proceeds via univariate numerical integration over time to maturity $\tau$. That integration can be simplified to averaging the elements of the sequence $\underline{\mathrm{f}}\left[x_{t, i}, 0\right], \ldots, \underline{\mathrm{f}}\left[x_{t, i}, \Delta \tau\right], \ldots, \underline{\mathrm{f}}\left[x_{t, i}, \tau_{k}\right]$, which is in turn obtained from the associated sequences of the closed form analytic calculations of $\mathrm{f}\left[x_{t, i}, \Delta \tau\right]$ and $\omega[\Delta \tau]$ and the functions $\Phi[\cdot]$ and $\phi[\cdot] .^{6}$

As detailed in Krippner (2013c) pp. 53-55, calculating the partial differential of $\underline{\mathrm{R}}\left[x(t), \tau_{k}\right]$ in equation 9 and making the substitution $x(t)=x_{t, i}$ in the result gives row $k$ of the Jacobian $H_{t, i}$ corresponding to $\underline{\mathrm{R}}\left[x_{t, i}, \tau_{k}\right]$, i.e.:

$$
H_{t, i, k}=\frac{1}{\tau_{k}} \int_{0}^{\tau_{k}} b_{0}^{\prime} \exp (-\tilde{\kappa} \tau) \cdot \Phi\left[\frac{\mathrm{f}\left[x_{t, i}, u\right]}{\omega(u)}\right]
$$

The numerical evaluations of $\Phi[\cdot]$ are already required for the measurement equation, i.e. the calculation of interest rates $\underline{\mathrm{R}}\left[x_{t, i}, \tau_{k}\right]$ from forward rates $\underline{\mathrm{f}}\left[x_{t, i}, \tau_{k}\right]$ in equation 11 . Therefore, no further implementations are required to obtain $H_{t, i, k}$.

\section{B-GATSM term structures and Jacobian}

B-GATSM bond prices for time to maturity $\tau$ may be defined generically as (see Krippner (2013c) p. 6):

$$
\underline{\mathrm{P}}[x(t), \tau]=\tilde{\mathbb{E}}_{t}\left[\exp \left(-\int_{0}^{\tau} \underline{\mathrm{r}}(t+u) \mathrm{d} u\right)\right]
$$

\footnotetext{
${ }^{5}$ A reference for this standard term structure relationship and others I use subsequently in the article is Filipović (2009) p. 7.

${ }^{6} \Phi[\cdot]$ does not actually have a mathematical closed form analytic solution, of course, but it is so well tabulated or approximated that it can be treated as having one. Also, it is more efficient in practice to calculate a single sequence of $\underline{\mathrm{f}}\left[x_{t, i}, \Delta \tau\right]$ out to the longest maturity $\tau_{K}$, and then simply use the results up to $\tau_{k}$ for the shorter maturities; see Krippner (2013c) pp. 52-53.
} 
where $\tilde{\mathbb{E}}_{t}$ is the risk-adjusted expectations operator with $\underline{\mathrm{r}}(t+u)=\max \{0, \mathrm{r}(t+u)\}$, where $\mathrm{r}(t+u)$ is as defined in equation 6 and $\max \{0, \cdot\}$ is the mechanism that imposes the ZLB.

B-GATSM interest rates $\underline{\mathrm{R}}[x(t), \tau]$ are obtained from $\underline{\mathrm{P}}[x(t), \tau]$ with a standard term structure relationship, i.e.:

$$
\underline{\mathrm{R}}[x(t), \tau]=-\frac{1}{\tau} \log \{\underline{\mathrm{P}}[x(t), \tau]\}
$$

From equation 9 , the row of the Jacobian $H_{t, i}$ corresponding to an interest rate $\underline{\mathrm{R}}\left[x_{t, i}, \tau_{k}\right]$ requires the partial differential of $\underline{\mathrm{R}}\left[x(t), \tau_{k}\right]$ with respect to $x(t)$, i.e.:

$$
\begin{aligned}
& \frac{\partial}{\partial x(t)} \underline{\mathrm{R}}\left[x(t), \tau_{k}\right]=\frac{\partial}{\partial x(t)}\left(-\frac{1}{\tau} \log \left\{\underline{\mathrm{P}}\left[x(t), \tau_{k}\right]\right\}\right) \\
& =-\frac{1}{\tau} \frac{\partial}{\partial x(t)} \log \left\{\underline{\mathrm{P}}\left[x(t), \tau_{k}\right]\right\} \\
& \langle\text { Chain rule }\rangle=-\frac{1}{\tau} \frac{\partial}{\partial \underline{\mathrm{P}}\left[x(t), \tau_{k}\right]} \log \left\{\underline{\mathrm{P}}\left[x(t), \tau_{k}\right]\right\} \cdot \frac{\partial}{\partial x(t)} \underline{\mathrm{P}}\left[x(t), \tau_{k}\right] \\
& =-\frac{1}{\tau} \frac{1}{\underline{\mathrm{P}}\left[x(t), \tau_{k}\right]} \cdot \frac{\partial}{\partial x(t)} \tilde{\mathbb{E}}_{t}\left[\exp \left(-\int_{0}^{\tau_{k}} \underline{\mathrm{r}}(t+u) \mathrm{d} u\right)\right] \\
& =-\frac{1}{\tau} \frac{1}{\underline{\mathrm{P}}\left[x(t), \tau_{k}\right]} \cdot \tilde{\mathbb{E}}_{t}\left\{\frac{\partial}{\partial x(t)} \exp \left(-\int_{0}^{\tau_{k}} \underline{\mathrm{r}}(t+u) \mathrm{d} u\right)\right\} \\
& \langle\text { Chain rule }\rangle=-\frac{1}{\tau} \frac{1}{\underline{\mathrm{P}}\left[x(t), \tau_{k}\right]} \cdot \tilde{\mathbb{E}}_{t}\left\{\frac{\partial}{\partial y} \exp [y] \cdot \frac{\partial}{\partial x(t)}\left[-\int_{0}^{\tau_{k}} \underline{\mathrm{r}}(t+u) \mathrm{d} u\right]\right\} \\
& \left\langle y=-\int_{0}^{\tau} \underline{\mathrm{r}}(t+u) \mathrm{d} u ; \frac{\partial}{\partial y} \exp [y]=\exp [y]\right\rangle \\
& =-\frac{1}{\tau} \frac{1}{\underline{\mathrm{P}}\left[x(t), \tau_{k}\right]} \cdot \tilde{\mathbb{E}}_{t}\left\{\exp [y] \cdot \frac{\partial}{\partial x(t)}\left[-\int_{0}^{\tau_{k}} \underline{\mathrm{r}}(t+u) \mathrm{d} u\right]\right\} \\
& =-\frac{1}{\tau} \frac{1}{\underline{\mathrm{P}}\left[x(t), \tau_{k}\right]} \cdot \tilde{\mathbb{E}}_{t}\left\{\exp [y] \cdot\left[-\int_{0}^{\tau_{k}} \frac{\partial}{\partial x(t)} \underline{\mathrm{r}}(t+u) \mathrm{d} u\right]\right\}
\end{aligned}
$$

The partial differential of $\underline{\mathrm{r}}(t+u)$ with respect to $x(t)$ is:

$$
\begin{aligned}
\frac{\partial}{\partial x(t)} \underline{\mathrm{r}}(t+u) & =\frac{\partial}{\partial x(t)} \max \{0, \mathrm{r}(t+u)\} \\
\underline{\mathrm{q}}(t+u) & =\left\{\begin{array}{cc}
0 & \text { if } \mathrm{r}(t+u) \leq 0 \\
\frac{\partial}{\partial x(t)} \mathrm{r}(t+u) & \text { if } \mathrm{r}(t+u)>0
\end{array}\right.
\end{aligned}
$$

From equation 6 with the normalization $\tilde{\theta}=0:^{7}$

$$
\mathrm{r}(t+u)=a_{0}+b_{0}^{\prime}\left\{\exp (-\tilde{\kappa} \tau) x(t)+\int_{t}^{t+u} \exp (-\tilde{\kappa}[u-v]) \sigma \mathrm{d} W(v)\right\}
$$

and therefore:

$$
\frac{\partial}{\partial x(t)} \mathrm{r}(t+u)=b_{0}^{\prime} \exp (-\tilde{\kappa} \tau)
$$

${ }^{7}$ Setting $\tilde{\theta}=0$ is a common (and convenient) normalization for the identification and estimation of GATSMs and shadow/ZLB-GATSMs; see, for example, Christensen and Rudebusch (2013a, b), Krippner (2013c), and $\mathrm{Wu}$ and Xia (2013). 
Substituting that result into equation 16 gives:

$$
\underline{\mathrm{q}}(t+u)=\left\{\begin{array}{cl}
0 & \text { if } \mathrm{r}(t+u) \leq 0 \\
b_{0}^{\prime} \exp (-\tilde{\kappa} \tau) & \text { if } \mathrm{r}(t+u)>0
\end{array}\right.
$$

and so the partial differential of $\underline{\mathrm{R}}\left[x(t), \tau_{k}\right]$ with respect to $x(t)$ result becomes:

$$
\frac{\partial}{\partial x(t)} \underline{\mathrm{R}}\left[x(t), \tau_{k}\right]=\frac{1}{\tau} \frac{1}{\underline{\mathrm{P}}\left[x(t), \tau_{k}\right]} \cdot \tilde{\mathbb{E}}_{t}\left\{\exp \left(-\int_{0}^{\tau_{k}} \underline{\mathrm{r}}(t+u) \mathrm{d} u\right) \cdot \int_{0}^{\tau_{k}} \underline{\mathrm{q}}(t+u) \mathrm{d} u\right\}
$$

Therefore, $H_{t, i, k}$ may be obtained by evaluating equation 20 directly using $\underline{\mathrm{r}}(t+u)$ and $\underline{\mathrm{q}}(t+u)$ generated with $x(t)=x_{t, i}$ in equation 17 .

As an example of applying the generic B-GATSM Jacobian principle in practice, the Monte Carlo implementation for a B-GATSM is:

$$
\widehat{\widehat{\mathrm{P}}}\left[x_{t, i}, \tau_{k}\right]=\frac{1}{J} \sum_{j=1}^{J} \exp \left[-\sum_{m=0}^{M} \underline{\mathrm{r}}_{j, m} \cdot \Delta \tau\right]
$$

where $\underline{\mathrm{r}}_{j, m}$ is obtained from the Black ZLB mechanism $\underline{\mathrm{r}}_{j, m}=\max \left\{0, \underline{\mathrm{r}}_{j, m}\right\}$, with $\underline{\mathrm{r}}_{j, m}=$ $a_{0}+b_{0}^{\prime} x_{j, m}$ and $x_{j, m}$ generated from a suitably discretized simulation of the state variable diffusion process under the $\mathbb{Q}$ measure, i.e.:

$$
x_{j, m}=x_{j, m-1}+\tilde{\kappa}\left[\tilde{\theta}-x_{j, m-1}\right] \Delta \tau+\sigma \sqrt{\Delta \tau} \tilde{\varepsilon}_{j, m}
$$

Regarding notation, $J$ is the number of simulations, $j$ is the index for each simulation, $m$ is the index for each step of the simulation, $M=\tau_{k} / \Delta \tau-1$ is the number of steps for each simulation, $\tilde{\varepsilon}_{j, m}$ are independent $\mathrm{N}(0,1)$ draws, and $x_{j, 0}=x_{t, i} \cdot{ }^{8}$

Row $k$ of the Jacobian $H_{t, i}$ is therefore:

$$
H_{t, i, k}=\frac{1}{\tau_{k}} \frac{1}{\underline{\underline{\mathrm{P}}}\left[x_{t, i}, \tau_{k}\right]} \cdot \frac{1}{J} \sum_{j=1}^{J} \exp \left[-\sum_{m=0}^{M-1} \underline{\underline{r}}_{j, m} \cdot \Delta \tau\right] \cdot \sum_{m=0}^{M-1} \underline{\mathrm{q}}_{j, m} \cdot \Delta \tau
$$

where:

$$
\underline{\mathrm{q}}_{j, m}=\left\{\begin{array}{cc}
0 & \text { if } \mathrm{r}_{j, m} \leq 0 \\
b_{0}^{\prime} \exp (-\tilde{\kappa} \tau) & \text { if } \mathrm{r}_{j, m}>0
\end{array}\right.
$$

The important point is that, because $\underline{\mathrm{q}}_{j, m}$ is an elementary transformation of $\mathrm{r}_{j, m}$, no further implementations are required to obtain the Jacobian beyond those already employed for the measurement equation.

\section{Conclusion}

This article shows that the principles for obtaining the Jacobian directly from the measurement equation calculations for the K-GATSM carries over to B-GATSMs. In particular,

\footnotetext{
${ }^{8}$ Analogous to the comment for the K-GATSM in footnote 4 , it is more efficient in practice to simulate single paths of $x_{j, m}$ out to the longest maturity $\tau_{K}$, and then use the results up to $\tau_{k}$ to obtain the results required for shorter maturities. Also, to maintain precise correspondence with the continuous time specification, the discretized $\tilde{\kappa}$, say $\tilde{\kappa}^{*}$, should actually be set to $\tilde{\kappa}^{*}=[I-\exp (-\tilde{\kappa} \Delta \tau)] / \Delta \tau$. However, because $\exp (-\tilde{\kappa} \Delta \tau) \simeq I-\tilde{\kappa} \Delta \tau$ the difference between $\tilde{\kappa}$ and $\tilde{\kappa}^{*}$ becomes practically negligible for suitably small values of $\Delta \tau$.
} 
only a single implementation per yield curve observation is required for the EKF, and combining this result with the faster B-GATSM implementations described in Priebsch (2013) or Krippner (2013a) should allow for much faster EKF estimations of multi-factor B-GATSMs.

More importantly, only a single implementation is also required to obtain the Jacobian per IEKF iteration, and the IEKF has been shown to provide more robust results than the EKF when estimating K-GATSMs (due to the high non-linearity in shadow/ZLBGATSMs; see Krippner (2013c) pp. 23-25). Faster B-GATSM implementations with several iterations of the IEKF should therefore provide more robust B-GATSM estimations than present EKF estimations. In addition, IEKF estimation should be faster than using the unscented Kalman filter estimation, as employed in Kim and Priebsch (2013), because the latter requires $2 N+1$ implementations for each observation of yield curve data.

\section{References}

Bauer, M. and G. Rudebusch (2013). Monetary policy expectations at the zero lower bound. Working Paper, Federal Reserve Bank of San Francisco.

Black, F. (1995). Interest rates as options. Journal of Finance 50, 1371-1376.

Cheridito, P., D. Filipović, and R. Kimmel (2007). Market price of risk specifications for affine models: theory and evidence. Journal of Financial Economics 83, 123-170.

Christensen, J. and G. Rudebusch (2013a). Estimating shadow-rate term structure models with near-zero yields. Working Paper, Federal Reserve Bank of San Francisco.

Christensen, J. and G. Rudebusch (2013b). Modeling yields at the zero lower bound: are shadow rates the solution? Presented at Term Structure Modeling at the Zero Lower Bound Workshop, Federal Reserve Bank of San Francisco, 11 October 2013.

Dai, Q. and K. Singleton (2002). Expectation puzzles, time-varying risk premia, and affine models of the term structure. Journal of Financial Economics 63, 415-441.

Duffee, G. (2002). Term premia and interest rate forecasts in affine models. Journal of Finance 57(1), 405-443.

Filipović, D. (2009). Term-Structure Models: A Graduate Course. Springer.

Jarrow, R. (2013). The zero-lower bound on interest rates: Myth or reality? Finance Research Letters 10, 151-156.

Kim, D. and M. Priebsch (2013). Estimation of multi-factor shadow rate term structure models. Presented at Term Structure Modeling at the Zero Lower Bound Workshop, Federal Reserve Bank of San Francisco, 11 October 2013.

Kim, D. and K. Singleton (2012). Term structure models and the zero bound: an empirical investigation of Japanese yields. Journal of Econometrics 170(1), 32-49.

Krippner, L. (2012). Modifying Gaussian term structure models when interest rates are near the zero lower bound. Discussion Paper, Reserve Bank of New Zealand DP2012/02.

Krippner, L. (2013a). Faster solutions for Black zero lower bound term structure models. Working Paper, Centre for Applied Macroeconomic Analyis 66/2013. 
Krippner, L. (2013b). Measuring the stance of monetary policy in zero lower bound environments. Economics Letters 118, 135-138.

Krippner, L. (2013c). A tractable framework for zero-lower-bound Gaussian term structure models. Discussion Paper, Reserve Bank of New Zealand.

Meucci, A. (2010). Review of statistical arbitrage, cointegration, and multivariate Ornstein-Uhlenbeck. Working Paper, SYMMYS.

Priebsch, M. (2013). Computing arbitrage-free yields in multi-factor Gaussian shadowrate term structure models. Working Paper, Federal Reserve Board 2013-63.

Richard, S. (2013). A non-linear macroeconomic term structure model. Working Paper, University of Pennsylvania.

Wu, C. and F. Xia (2013). Measuring the macroeconomic impact of monetary policy at the zero lower bound. Working Paper. 